\title{
Analysis of Occlusal Stresses Transmitted to the Inferior Alveolar Nerve by Multiple Threaded Implants
}

\author{
G. Sammartino, ${ }^{*}$ Hom-Lay Wang,${ }^{\dagger}$ R. Citarella,${ }^{\ddagger}$ M. Lepore,${ }^{\dagger}$ and G. Marenzi ${ }^{*}$
}

Background: Potential nerve injury or loss of sensation can occur after mandibular implant placement or loading. To avoid this type of damage, it is critical to determine the proper distance from implants to the mandibular nerve. Hence, the purpose of this study is to use biomechanical analyses to determine the safe distance from multiple implants to the inferior alveolar nerve.

Methods: Using the boundary element method, a numerical mandibular model was designed to simulate a mandibular segment containing multiple threaded fixtures. This model allows assessment of the pressure, as induced by occlusal loads, on the trigeminal nerve. Such pressure distribution was evaluated against different distances from the fixtures to the mandibular canal, against the possible lack of the central fixture in a three-abutment configuration, and against different levels of implant osseointegration. All the simulations considered a canal that is orthogonal to the implant axis.

Results: Nerve pressure increased quickly when the implant-canal distance decreased in the range studied. Lack of the central implant to support the central abutment caused major increases in nerve pressure.

Conclusions: This study suggests a minimal implant-canal distance of $1 \mathrm{~mm}$ to prevent inferior alveolar nerve damage caused by three connected implants. For clinical safety, an additional $0.5 \mathrm{~mm}$ is recommended as a cushion, so a 1.5 mm minimal distance should be planned to avoid potential nerve injury. J Periodontol 2013;84:1655-1661.

\section{KEY WORDS}

Dental implants; dental stress analysis; dysesthesia; mandibular nerve; occlusal force; trigeminal nerve.

\footnotetext{
* Department of Neurosciences, Reproductive and Odontostomatological Sciences, School of Medicine, University "Federico II," Naples, Italy.

† Department of Periodontics and Oral Medicine, University of Michigan, Ann Arbor, MI.

‡ Department of Industrial Engineering, University of Salerno, Fisciano (SA), Italy.
}

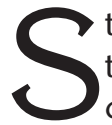
tudies have shown changes in trigeminal sensibility after mandibular surgical treatment ${ }^{1-4}$ and implant placement. ${ }^{5-11}$ Placement of dental implants in the mandible can cause an insult to the nervous structures and transitory or irreversible alterations of inferior nerve functionality. ${ }^{7}$ Paresthesia and dysesthesia following implant loading, due to occlusal force compression on the nerve, have been reported. ${ }^{12}$ To prevent this nerve injury, assessment of the mandibular canal position and selection of a proper implant size, as well as the proper positioning of implants, are needed. ${ }^{13,14}$ Studies have suggested the favorable positioning of a fixture with respect to adjacent natural teeth or, in more complex rehabilitations, the distance between fixtures to achieve the best esthetic result ${ }^{15}$ and an optimal distribution of occlusal forces. ${ }^{16}$ However, limited evidence exists with regard to the proper distance from implant to mandibular nerve that can ensure the implant's integrity as well as physiologic activity. ${ }^{17}$ It is the opinion of the authors of the present study that this proper distance should be deduced not only from the evaluation of clinical data (retrospective study), but also from biomechanical analysis. 18,19 Therefore, using boundary element method (BEM), a numeric methodology was created to simulate a mandibular segment containing multiple fixtures, so that the mechanical stresses induced by the

doi: 10.1902/jop.2013.120611 
occlusal loads on the mandibular canal could be properly assessed.

\section{MATERIALS AND METHODS}

Based on 123 mandibular computer tomography scans obtained from the University of Naples "Federico II," the size and bone density of the anatomic structures (cortical bone, cancellous bone, cortical bone surrounding the mandibular canal) were calculated (using Hounsfield values), and then a numeric model for stress testing was developed. ${ }^{17}$ The average density and dimensional values of each examined anatomic structure were identified and then reproduced in this simulated model (see previous paper ${ }^{17}$ ). The mandibular numeric model was created using commercial software ${ }^{\S}$ based on BEM, a numeric methodology that is well suited for elasticstatic analysis. The implant used in the design was a conic threaded fixture of $4.5-\mathrm{mm}$ diameter and 11 mm length.

$\mathrm{BEM}$ methodology is certainly more accurate for linear analysis but less versatile than finite element method (FEM), ${ }^{20}$ especially in the related area of a complex geometry such as the threaded implant, ${ }^{16}$ where strong stress gradients are to be captured. Moreover, with BEM, it is easier (compared with FEM) to mesh complex geometries, such as the thread of the implant body, which in most numeric studies is not represented in continuous helical characteristics but only approximated as axialsymmetric independent rings. ${ }^{16}$

The mandibular segment was modeled with a mesh of $\approx 5,000$ linear elements, with the fixture connected to a prosthesis abutment on which the axial and lateral loads were applied (Fig. 1A). It is widely agreed to be more accurate to model bone material constants as orthotropic ${ }^{21}$ rather than isotropic; hence both the cortical and cancellous bone were modeled as transversely isotropic. The elastic behavior of transversely isotropic materials can be fully characterized by five elastic moduli: $E_{1}, E_{3}, v_{12}, v_{31}$, and $G_{31}$, with the remaining moduli obtained from the relations $E_{1}=E_{2}, \nu_{23}=\nu_{13}=$ $\nu_{31} * E_{1} / E_{3}, G_{12}=E_{2} /\left[2\left(1+v_{12}\right)\right]$, and $G_{23}=G_{31}$. The values adopted in this work are taken from the literature. ${ }^{17}$

As in a previous study by the present authors, ${ }^{17}$ the trigeminal nerve was modeled as isotropic, with Young modulus $E=1.3$ megapascals $(\mathrm{MPa})$ and Poisson ratio $v=0.4$. The metallic implant parts were modeled as isotropic, with $E=120,000 \mathrm{MPa}$ and $v=$ 0.3 .

To calculate the pressure on the nerve, a nonlinear BEM contact analysis was performed, involving the interface between nerve and canal, with a null clearance imposed between the nerve and the
A
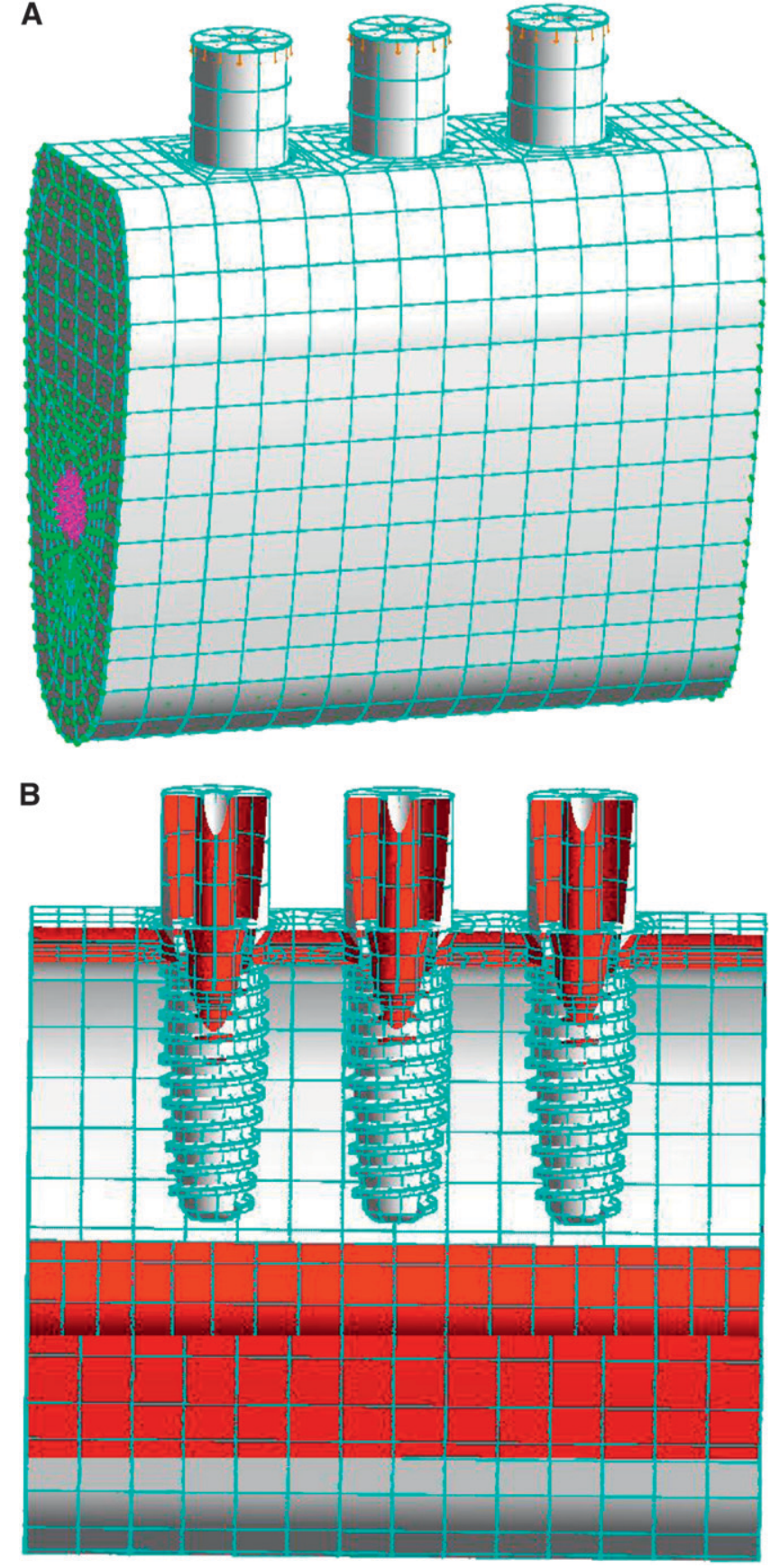

Figure I.

A) BEM numeric model of the mandibular segment and implants, with highlight of the axial applied load, mesh, and boundary conditions. B) BEM numeric model of the mandibular segment and implants; internal view with highlight of the canal, the separation between cortical and spongy bone, and the detail of the abutment-implant connection. 
surrounding canal structures (this is the worst case, because in reality a minimum clearance is available to the nerve in the canal). The global load, applied along the implant axis, was equal to $300 \mathrm{~N}$ but split on three equally spaced implants (implant pitch $=7$ $\mathrm{mm}$ ) in such a way that a load equal to $100 \mathrm{~N}$ was applied on each abutment (Fig. 1A). The choice of these forces corresponds to physiologic occlusal loads during chewing and swallowing. ${ }^{22}$

The implants considered are disconnected or connected by two abutments made of cobalt-chrome alloyll (99.8\% gold), with Young modulus $E=77 \mathrm{GPa}$ and Poisson ratio $v=0.42$. The pressure distribution induced on the underlying nervous structure was evaluated against different distances between the fixture bottom and the upper part of the mandibular canal $\left(d_{1}=1.0 \mathrm{~mm}, d_{2}=1.5 \mathrm{~mm}\right)$. Moreover, all the simulations considered a canal that is orthogonal to the implant axis (Fig. 1B).

A sensitivity analysis was also done to show the effect on nerve pressure levels of four similar prosthetic rehabilitations: 1) three disconnected crowns supported by three different osseointegrated fixtures (Fig. 2A); 2) three connected crowns supported by three different osseointegrated implants (Fig. 2B); 3) three connected crowns supported by two different osseointegrated implants (the lateral ones) (Fig. 2C); and 4 ) three connected crowns supported by partially osseointegrated implants with, in particular, a complete lack of osseointegration between central implant and cortical bone (whereas osseointegration is complete for the lateral implants) (see Fig. 2B). The variations of bone stiffness as a function of bone density were calculated considering a cubic dependence of stiffness versus density. ${ }^{23}$

\section{RESULTS}

To understand which bone part is critical for fixture load absorption, some simulations were done considering the possibility of cortical bone resorption around the implant collar and the fixture-canal distance 1.0 or $1.5 \mathrm{~mm}$.

In Figure 3, the BEM contour plots show the pressure on the nervous structure in the four considered configurations and at a distance of $1 \mathrm{~mm}$ between the fixture and the upper part of the mandibular canal. When the distance is increased from 1.0 to $1.5 \mathrm{~mm}$, the nerve pressure decreases: the maximum pressure on the nerve is reported in Figure 4 for the four considered groups and the two distances between implant and nerve.

The weakening of the implant connection to the cortical bone (case 4) was shown in a moderate increase (from $3 \times 10^{-2}$ to $3.17 \times 10^{-2} \mathrm{MPa}$ ) of the maximum nerve pressure with respect to case 2 (Figs. 3B, 3D, and 4). Increments of nerve pressure become more consistent (from $3 \times 10^{-2}$ to $3.34 \times$ $10^{-2} \mathrm{MPa}$ ) when the central implant is not introduced at all (case 3), providing the most critical condition among those analyzed (Figs. $3 \mathrm{C}$ and 4).

The configuration with three disconnected implants (case 1) is reported to provide an initial reference condition but, considering the loading condition analyzed, is less realistic, because a uniform distribution of the mastication load on the three considered implants is possible only when such implants are connected to each other.

Although the minimal distance for the safety margin is $1.0 \mathrm{~mm}, 0.5 \mathrm{~mm}$ has been added to the recommendation as a safety cushion. This additional 0.5-mm distance can minimize any measuring error that occurs in the calculation (Fig. 4). Hence, the recommended distance would be at least $1.5 \mathrm{~mm}$.

\section{DISCUSSION}

Peripheral neuropathies with conduction block can have different etiologies: physical (electrical, thermal, percussion, compression injures, radiation-induced), toxic (acting on either axon or myelin), more rarely ischemic (arteriopathy or vasculitis), or frequently inflammatory (acute or chronic, primary or secondary) or related to dysimmune states. ${ }^{24}$ Two main factors may be involved in producing axonal transport block during compression of a peripheral nerve: ischemia (due to occlusion of vasa vasorum) and mechanical deformation of the nerve fibers. ${ }^{25}$ It is apparent that ischemia and deformations can be in relation to chronic and acute compression disorders, respectively. ${ }^{26}$ Time and degree of compression, type (A1, A2, C, myelinated or not), and size (multifascicular or not) of the fibers influence a critical pressure for each nerve where the fiber viability is acutely jeopardized. ${ }^{27}$ Many investigators have confined their attention to isolated nerves, and their methods have been so various that results are not strictly comparable. Rydevik et al. ${ }^{25}$ reported that in rabbit vagus nerve, a pressure of $50 \mathrm{mmHg}$ applied for 2 hours may induce a blockage of axonal transport that is reversible within 1 day. A pressure of 200 or $400 \mathrm{mmHg}$ applied for 2 hours similarly induced a block of axonal transport persisting up to at least 2 and 3 days, respectively, after the compression. ${ }^{25}$ Fern and Harrison ${ }^{26}$ reported that a pressure of 70 $\mathrm{mmHg}$ induced conduction block in cat sciatic nerve in an average of 56 minutes, with the slow-conducting axons (unmyelinated) tending to fail before the faster-conducting axons. In contrast, a pressure of $250 \mathrm{mmHg}$ produced conduction block in an average of 35 minutes, and the fast-conducting axons (myelinated) tended to block first. ${ }^{26}$ In another work,

\| Keramit, NOBIL-METAL, Villafranca d'Asti, Italy. 

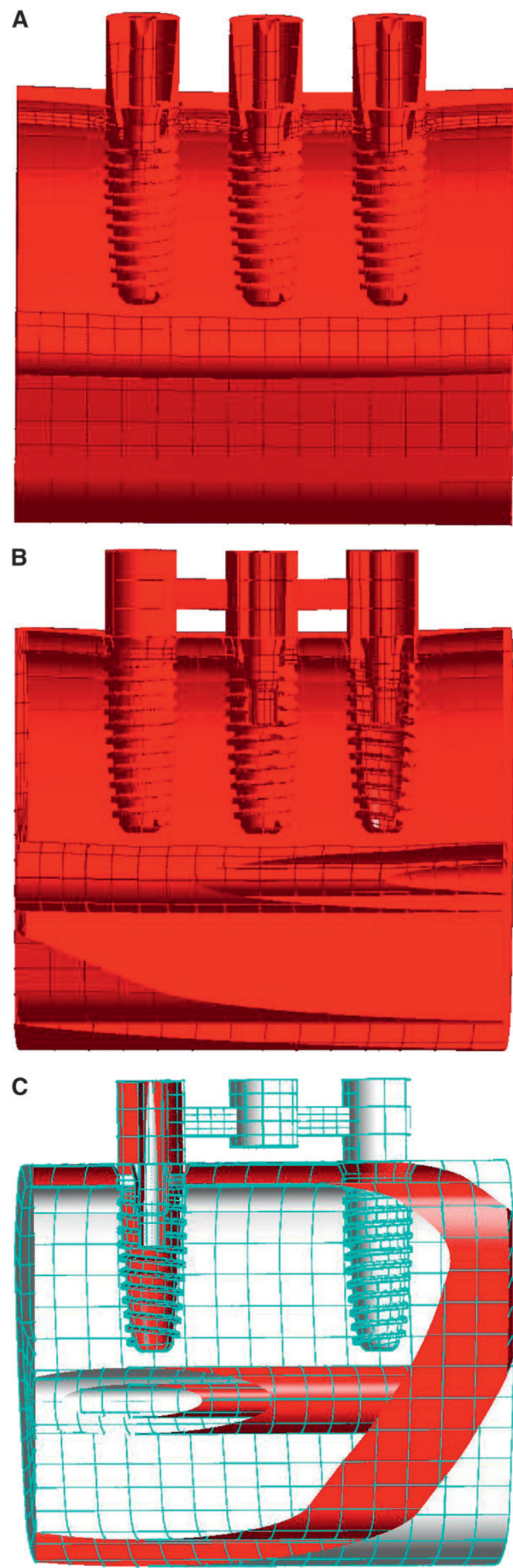

Fern and Harrison ${ }^{28}$ showed that in frog sciatic nerve, a pressure of $250 \mathrm{mmHg}$ is able to produce a first block of the faster-conducting axons, whereas a pressure of $750 \mathrm{mmHg}$ is able to produce a first block of the slower-conducting myelinated axons.

Bentley and Schlapp ${ }^{29}$ showed that a pressure of $\leq 100 \mathrm{mmHg}$ acting for 2 to 3 hours does not produce a block of peripheral nerve. At a pressure of 120 $\mathrm{mmHg}$, there is some interference with conduction, and with a pressure varying between 130 and 200 $\mathrm{mmHg}$, a block is first apparent in about 40 minutes and is complete (or almost so) in 2 to 3 hours. Recovery from complete block does not occur within 3 hours of pressure release, but in one experiment, observed for 13 hours, conduction recovered in about one-third of the fibers. There is a critical value of $\approx 130 \mathrm{mmHg}$ for the production of nerve block if the pressure is applied for a period of 2 to 3 hours. ${ }^{29}$ The inferior alveolar nerve and the trigeminal system have an ultrastructural propensity to compressive injury, because the latter has the greatest proportion of myelinated axons in the entire somatosensory system. ${ }^{30}$ Information about trigeminal reaction to local applied pressure is shown by the compressive technique performed with stents applied transcutaneously, close to the oval foramen used to control neuralgias of the trigeminal nerve. ${ }^{31-36}$

Pressures $>100$ to $200 \mathrm{mmHg}$ applied for 30 to 60 seconds to the trigeminal nerve can cause a block of nervous transmission and, in the neuralgias, solve the pain problem. ${ }^{30,34}$ Studies have reported alteration of mandibular nerve sensibility following implant placement. ${ }^{5-11}$ This alteration can be either transitory or non-reversible depending on the severity of nerve damage. ${ }^{8}$ Functional alteration of mandibular nerve can be caused by excessive drilling pressure, soft tissue injury during surgery, post-surgery edema or hematoma, or implant impingement on the mandibular nerve. ${ }^{1-4,37}$

When the implant is placed too close to the inferior alveolar canal, mechanical stress to the underlying canal can be induced that often results in alteration of nerve function. ${ }^{12}$ Studies ${ }^{7,11,13}$ have suggested that a safe distance is required to avoid nerve injury; however, biomechanical consideration was not addressed in those reports. Although some surgical solutions to nerve injury have been proposed, ${ }^{12}$ it is advisable to plan the implant position before implant insertion to ensure a safe distance between implant fixtures and the mandibular canal.

Figure 2.

A) Deformed plot (scale factor 70) in the case of lack of connection among the three implants (internal view). B) Deformed plot (scale factor 70) with three connected and osseointegrated implants (internal view).

C) Configuration in the case of lack of the central implant (internal view). 

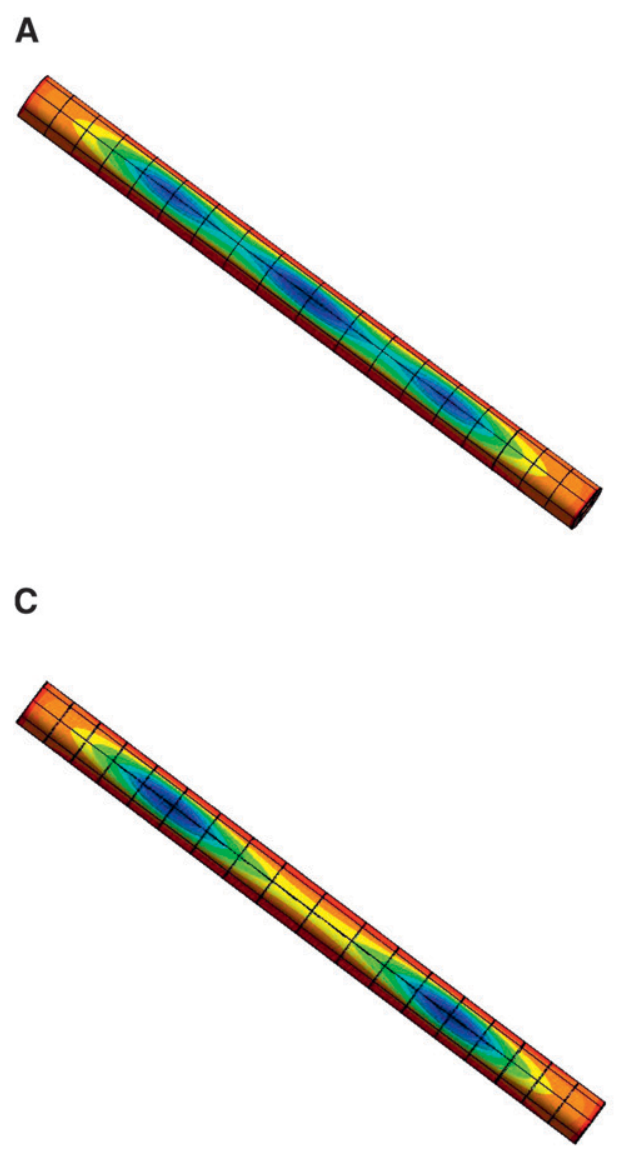
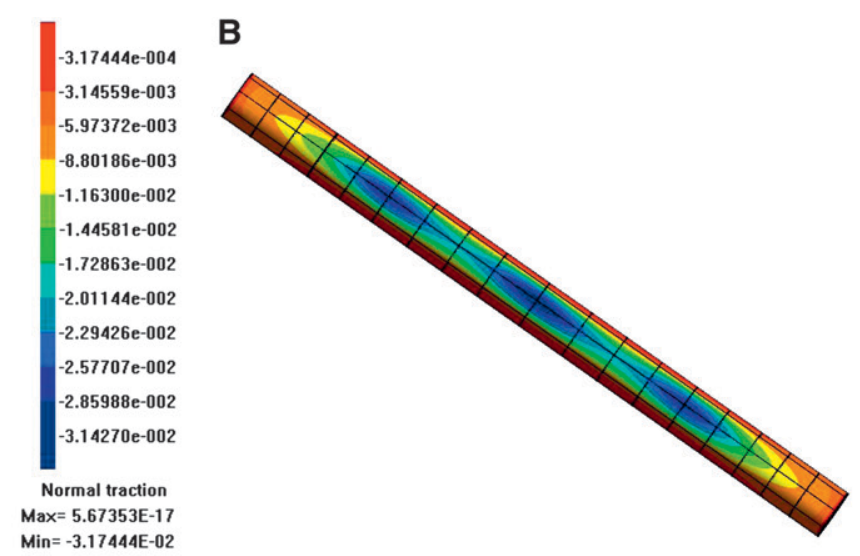

D

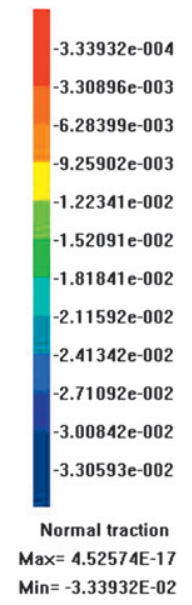

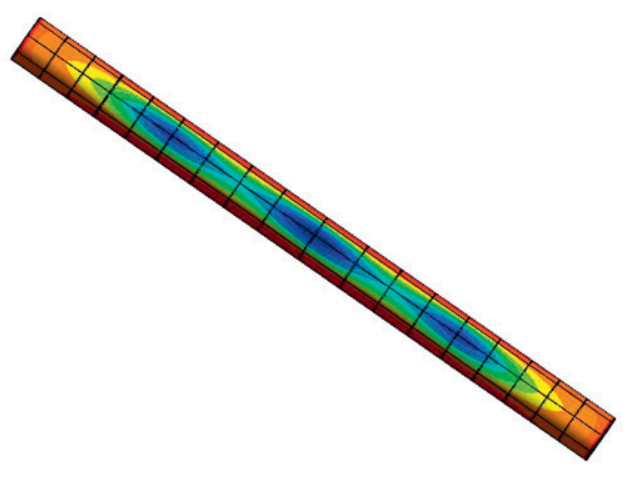

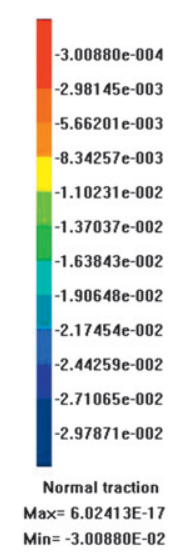

Figure 3.

A) Nerve pressure (MPa) in the case of lack of connection among the three implants and distance between implant and canal equal to $1.0 \mathrm{~mm}$. B) Nerve pressure with connected and osseointegrated implants and distance between implant and canal equal to $1.0 \mathrm{~mm}$. C) Nerve pressure in the case of lack of the central implant and distance between implant and canal equal to $1.0 \mathrm{~mm}$. D) Nerve pressure with connected but partially osseointegrated implants and distance between implant and canal equal to $1.0 \mathrm{~mm}$.

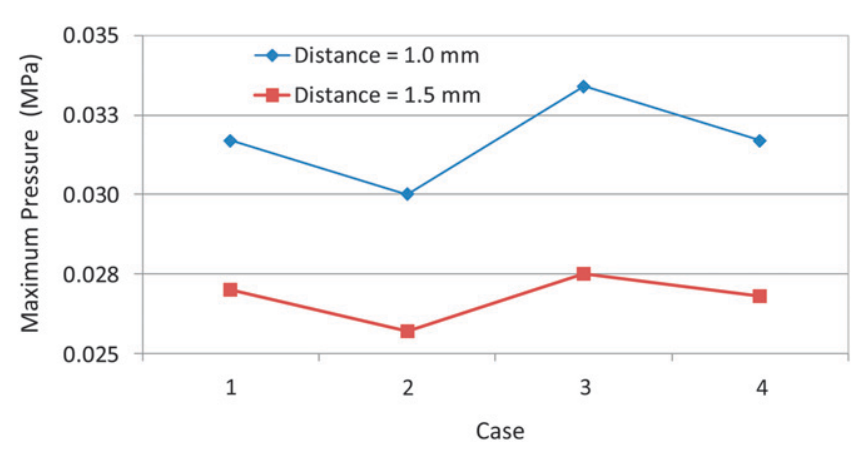

Figure 4.

Maximum pressure (MPa) on the nerve against the four considered cases and the two distances between implant and nerve.
This investigation allows an evaluation of the pressures that three conic threaded fixtures, undergoing a functional load, are able to transmit to the underlying bony structures. Data from this investigation showed the sensitivity of nerve pressure against effective introduction of the central implant (rather than simply connecting the central abutment to the lateral implants), with particular reference to a distance between implant (bottom part) and canal (upper part) of 1.0 or $1.5 \mathrm{~mm}$. The numeric analyses showed how nerve peak pressure increased when the implant-to-canal distance decreased and when the central implant was missing.

Furthermore, this study assesses the effects on the nerve of a total cortical resorption around the central implant. When a central implant is missing or osseointegration is lacking, these data suggested that with a multiple fixture-canal distance of $1 \mathrm{~mm}$, occlusal forces transmitted by an implant can generate enough stress to the trigeminal nerve to trigger 
mandibular sensibility alterations. The maximum calculated pressure values are very similar to the pressures used in neurosurgery. However, since the high force produced during occlusal contact persists only for an average of 115 milliseconds, ${ }^{22}$ these contacts are not able to alter the trigeminal functionality, also according to clinical data. ${ }^{6-11}$ Hence, the implication is that a multiple fixture-canal distance of $1 \mathrm{~mm}$ is acceptable in presurgical planning when considering three connected implants (with or without osseointegration) or two fully osseointegrated implants with three abutments. For a clinical safety cushion, the recommendation was raised by an additional $0.5 \mathrm{~mm}$, so $1.5 \mathrm{~mm}$ minimal distance should be planned to avoid any potential nerve injury during implant placement or after loading.

\section{CONCLUSIONS}

This study suggests that a minimal distance between implant and canal should be at least $1.0 \mathrm{~mm}$ to prevent damage caused by three connected implants to the underneath inferior alveolar nerve when biomechanical loading is taken into consideration. This implies that a distance $d=1.0 \mathrm{~mm}$ (plus a margin needed to take into account the loss of precision due to measurement error during implant placement) is an acceptable reference distance in presurgical planning when using three connected implants (with or without complete osseointegration) or two fully osseointegrated implants with three abutments.

\section{ACKNOWLEDGMENTS}

The authors do not have any financial interests, either directly or indirectly, in the products or information listed in the paper. The authors report no conflicts of interest related to this study.

\section{REFERENCES}

1. de Koomen HA. A prosthetic view on vestibuloplasty with free mucosal graft. Int J Oral Surg 1977;6: 38-41.

2. Bailey PH, Bays RA. Evaluation of long-term sensory changes following mandibular augmentation procedures. J Oral Maxillofac Surg 1984;42:722-727.

3. Kipp DP, Goldstein BH, Weiss WW Jr. Dysesthesia after mandibular third molar surgery: A retrospective study and analysis of 1,377 surgical procedures. J Am Dent Assoc 1980;100:185-192.

4. Simpson HE. Injuries to the inferior dental and mental nerves. J Oral Surg (Chic) 1958;16:300-305.

5. Walton JN. Altered sensation associated with implants in the anterior mandible: A prospective study. $J$ Prosthet Dent 2000;83:443-449.

6. Ellies LG. Altered sensation following mandibular implant surgery: A retrospective study. J Prosthet Dent 1992;68:664-671.

7. Wismeijer D, van Waas MA, Vermeeren JI, Kalk W. Patients' perception of sensory disturbances of the mental nerve before and after implant surgery: A prospective study of 110 patients. Br J Oral Maxillofac Surg 1997;35:254-259.

8. Rubenstein JE, Taylor TD. Apical nerve transection resulting from implant placement: A 10-year follow-up report. J Prosthet Dent 1997;78:537-541.

9. Ellies LG, Hawker PB. The prevalence of altered sensation associated with implant surgery. Int $J$ Oral Maxillofac Implants 1993;8:674-679.

10. Gregg JM. Neuropathic complications of mandibular implant surgery: Review and case presentations. Ann R Australas Coll Dent Surg 2000;15:176-180.

11. Bartling R, Freeman K, Kraut RA. The incidence of altered sensation of the mental nerve after mandibular implant placement. J Oral Maxillofac Surg 1999;57: 1408-1412.

12. Levitt DS. Apicoectomy of an endosseous implant to relieve paresthesia: A case report. Implant Dent 2003; 12:202-205.

13. Greenstein G, Tarnow D. The mental foramen and nerve: Clinical and anatomical factors related to dental implant placement: A literature review. J Periodontol 2006;77:1933-1943.

14. Santler G, Kärcher H, Ruda C. Indications and limitations of three-dimensional models in cranio-maxillofacial surgery. J Craniomaxillofac Surg 1998;26:11-16.

15. Kois JC. Predictable single tooth peri-implant esthetics: Five diagnostic keys. Compend Contin Educ Dent 2001;22:199-206, quiz 208.

16. Citarella R, Armentani E, Caputo F, Lepore M. Stress analysis of an endosseus dental implant by BEM and FEM. Open Mech Eng J 2012;6:115-124.

17. Sammartino G, Marenzi G, Citarella R, Ciccarelli R, Wang H-L. Analysis of the occlusal stress transmitted to the inferior alveolar nerve by an osseointegrated threaded fixture. J Periodontol 2008;79:1735-1744.

18. Citarella R, Armentani E, Caputo F, Naddeo A. FEM and BEM Analysis of a human mandible with added temporomandibular joints. Open Mech Eng J 2012;6: 100-114.

19. Armentani E, Caputo F, Citarella R. FEM sensitivity analyses on the stress levels in a human mandible with a varying ATM modelling complexity. Open Mech Eng J 2010;4:8-15.

20. Brebbia CA, Telles JC, Wrobel LC. Boundary Element Techniques. Berlin: Springer Verlag; 1984:1-100.

21. Rho JY, Hobatho MC, Ashman RB. Relations of mechanical properties to density and CT numbers in human bone. Med Eng Phys 1995;17:347-355.

22. Gibbs $\mathrm{CH}$, Mahan PE, Lundeen HC, et al. Occlusal forces during chewing and swallowing as measured by sound transmission. J Prosthet Dent 1981;46:443-449.

23. Wirtz DC, Schiffers N, Pandorf T, Radermacher K, Weichert D, Forst R. Critical evaluation of known bone material properties to realize anisotropic FE-simulation of the proximal femur. J Biomech 2000;33:1325-1330.

24. Battista AF, Alban E. Effect of graded ligature compression on nerve conduction. Exp Neurol 1983;80: $186-194$.

25. Rydevik B, McLean WG, Sjöstrand J, Lundborg G. Blockage of axonal transport induced by acute, graded compression of the rabbit vagus nerve. J Neurol Neurosurg Psychiatry 1980;43:690-698.

26. Fern R, Harrison PJ. The contribution of ischaemia and deformation to the conduction block generated by compression of the cat sciatic nerve. Exp Physiol 1994;79:583-592. 
27. Lundborg G, Gelberman RH, Minteer-Convery M, Lee YF, Hargens AR. Median nerve compression in the carpal tunnel - Functional response to experimentally induced controlled pressure. J Hand Surg Am 1982;7: 252-259.

28. Fern R, Harrison PJ. The effects of compression upon conduction in myelinated axons of the isolated frog sciatic nerve. J Physiol 1991;432:111-122.

29. Bentley FH, Schlapp W. The effects of pressure on conduction in peripheral nerve. J Physiol 1943;102: 72-82.

30. Donoff RB. Nerve regeneration: Basic and applied aspects. Crit Reu Oral Biol Med 1995;6:18-24.

31. Lee ST, Chen JF. Percutaneous trigeminal ganglion balloon compression for treatment of trigeminal neuralgia - Part I: Pressure recordings. Surg Neurol 2003; 59:63-66, discussion 66-67.

32. Lee ST, Chen JF. Percutaneous trigeminal ganglion balloon compression for treatment of trigeminal neuralgia, Part II: Results related to compression duration. Surg Neurol 2003;60:149-153, discussion 153-154.

33. Brown JA, Pilitsis JG. Percutaneous balloon compression for the treatment of trigeminal neuralgia: Results in 56 patients based on balloon compression pressure monitoring. Neurosurg Focus 2005; 18:E10.
34. Brown JA, McDaniel MD, Weaver MT. Percutaneous trigeminal nerve compression for treatment of trigeminal neuralgia: Results in 50 patients. Neurosurgery 1993;32:570-573.

35. Brown JA, Hoeflinger B, Long PB, et al. Axon and ganglion cell injury in rabbits after percutaneous trigeminal balloon compression. Neurosurgery 1996;38: 993-1003, discussion 1003-1004.

36. Belber CJ, Rak RA. Balloon compression rhizolysis in the surgical management of trigeminal neuralgia. Neurosurgery 1987;20:908-913.

37. Park YT, Kim SG, Moon SY. Indirect compressive injury to the inferior alveolar nerve caused by dental implant placement. J Oral Maxillofac Surg 2012;70:e258e259.

Correspondence: Prof. Gilberto Sammartino, Department of Odontostomatological and Maxillofacial Sciences, Division of Oral Surgery, Faculty of Medicine, University of Naples "Federico II," Via Pansini 5, Ed. 14, 80131, Naples, Italy. Fax: +39 81 7462118; e-mail: gilberto.sammartino@ unina.it.

Submitted October 9, 2012; accepted for publication December 19, 2012. 